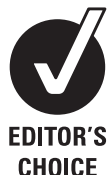

CHOICE

\title{
Utility of multispectral imaging in automated quantitative scoring of immunohistochemistry
}

\author{
Christopher Fiore, ${ }^{1,2}$ Dyane Bailey, ${ }^{1,2}$ Niamh Conlon, ${ }^{3}$ Xiaogiu Wu, ${ }^{1,2}$ Neil Martin, ${ }^{4}$ \\ Michelangelo Fiorentino, ${ }^{1,2,5}$ Stephen Finn, ${ }^{1,2,3}$ Katja Fall, ${ }^{6,7}$ Swen-Olof Andersson, ${ }^{7}$ \\ Ove Andren, ${ }^{7}$ Massimo Loda, ${ }^{1,2}$ Richard Flavin ${ }^{1,2,3}$
}

${ }^{1}$ Center for Molecular Oncologic Pathology, Dana Farber Cancer Institute, Boston, Massachusetts, USA ${ }^{2}$ Brigham and Women's Hospital, Harvard Medical School, Boston, Massachusetts, USA

${ }^{3}$ Department of Pathology, Trinity College, Dublin, Ireland ${ }^{4}$ Department of Radiation Oncology, Harvard Radiation Oncology Program, Boston,

Massachusetts, USA ${ }^{5}$ Pathology Unit, Addarii Institute, S Orsola-Malpighi Hospital, Bologna, Italy ${ }^{6}$ School of Health and Medical Sciences, Örebro University, Orebro, Sweden

${ }^{7}$ Harvard School of Public Health, Boston, MA, USA

\section{Correspondence to}

Dr Richard Flavin, Department of Pathology, St James's Hospital, James's Street, Dublin 8 , Ireland;

flavinrichard@gmail.com

Accepted 6 February 2012 Published Online First 23 March 2012

\section{ABSTRACT \\ Background Automated scanning devices and image analysis software provide a means to overcome the limitations of manual semiquantitative scoring of immunohistochemistry. Common drawbacks to automated imaging systems include an inability to classify tissue type and an inability to segregate cytoplasmic and nuclear staining.}

Methods Immunohistochemistry for the membranous marker $\alpha$-catenin, the cytoplasmic marker stathmin and the nuclear marker Ki-67 was performed on tissue microarrays (TMA) of archival formalin-fixed paraffinembedded tissue comprising 471 ( $\alpha$-catenin and stathmin) and 511 (Ki-67) cases of prostate adenocarcinoma. These TMA were quantitatively analysed using two commercially available automated image analysers, the Ariol SL-50 system and the Nuance system from CRi. Both systems use brightfield microscopy for automated, unbiased and standardised quantification of immunohistochemistry, while the Nuance system has spectral deconvolution capabilities. Results Overall concordance between scores from both systems was excellent $(r=0.90 ; 0.83-0.95)$. The software associated with the multispectral imager allowed accurate automated classification of tissue type into epithelial glandular structures and stroma, and a single-step segmentation of staining into cytoplasmic or nuclear compartments allowing independent evaluation of these areas. The Nuance system, however, was not able to distinguish reliably between tumour and non-tumour tissue. In addition, variance in the labour and time required for analysis between the two systems was also noted.

Conclusion Despite limitations, this study suggests some beneficial role for the use of a multispectral imaging system in automated analysis of immunohistochemistry.

Over the years, identifying tissue biomarkers has been the focus of a large body of research to help classify cancers for diagnostic, prognostic and therapeutic purposes. This ability to subclassify cancers is dependent on the means of quantifying the amount of biomarker present in tissue. Immunohistochemistry is a technique frequently used to visualise protein present in tissue. Traditionally, histopathologists have scored immunohistochemistry manually; however, this can be labour and time intensive, semiquantitative and subject to observer bias. Comparisons of scoring methods by different pathologists have shown relatively accurate reproducibility of scores for the intensity of staining but a pronounced lack of reliability for the extent of staining. ${ }^{1}$ Tissue microarrays (TMA) allow immunohistochemical analysis of large numbers of tumour samples in a high-throughput manner. Potentially up to 400 tissue cores representing more than 130 tumours in triplicate can be placed onto one TMA block. While this allows for increased speed of analysis, it is still inefficient when scored semiquantitatively by pathologists. In addition, it can also be difficult to set an appropriate quantitative scale that can be accurately followed by the observer. ${ }^{2}$ As such, automated scanning devices and image analysis software coupled with TMA may provide a means to overcome such limitations. ${ }^{3}$

Various methods of automated scoring of immunohistochemistry have proved to be an effective substitute for manual scoring. The Ariol SL-50 system (Genetix, San Jose, California, USA) is a widely used platform for automated immunohistochemical analysis. ${ }^{2}{ }^{4}$ Quantitative scores using this system show very good correlation with semiquantitative manual scores in both small and large study cohorts, with comparable patient outcome data generated from both systems of analysis. ${ }^{4-6}$ Furthermore, these and other studies have indicated that automated quantitative systems are an excellent substitute for manual semiquantitative scoring by a pathologist. ${ }^{5-9}$ Of importance, automation reduces the labour and time required to score immunohistochemically, and can also produce improvements in scoring such as the reproducibility and reliability of the score for the total area of staining. ${ }^{4}$ Indeed, automated systems may help lead to a standardised system of immunohistochemical scoring and reduce the dependence of scores on arbitrary thresholds. ${ }^{10}$

However, computational image analysis is not without problems. Tumour tissue may be quite heterogenous morphologically, comprising both epithelial and stromal compartments; this can be difficult to analyse consistently using existing computer software programs. Some tumours, such as prostatic adenocarcinoma, can cause special problems with analysis due to the infiltrative nature of malignant glands among normal epithelial tissue and prostatic intra-epithelial neoplasia coexisting adjacent to invasive adenocarcinoma. The major limitation of some automated systems is an inability to classify the tissue type automatically. This necessitates manually marking tumour 
Table 1 Study cohort characteristics (Swedish Watchful Waiting Cohort I: stained for stathmin and Ki67

\begin{tabular}{ll}
\hline No of patients & 571 \\
& Mean age, years \\
Age at diagnosis & $73.08(51-91)$ \\
Gleason score & $n(\%)$ \\
4 & $9(1.6)$ \\
5 & $14(2.5)$ \\
6 & $224(39.2)$ \\
7 & $203(35.6)$ \\
8 & $53(9.3)$ \\
9 & $59(10.3)$ \\
10 & $9(1.6)$ \\
$\%$ Chips with cancer & \\
$<5$ & $191(33.5)$ \\
$5-25$ & $240(42.0)$ \\
$26-50$ & $63(11.0)$ \\
$>50$ & $77(13.5)$ \\
t-Stage & \\
T1a & $191(33.5)$ \\
T1b & $380(66.6)$ \\
Cause of death & \\
PC death & $102(17.9)$ \\
Non-PC death & $346(60.6)$ \\
Still alive & $73(12.8)$ \\
\hline
\end{tabular}

$\mathrm{PC}$, prostate cancer.

areas for analysis for each core on a TMA. The software programs are also imperfect at segmenting tissue into nuclear and cytoplasmic areas, especially when there is strong intense immunohistochemical staining. Furthermore, there may be problems with colour pattern recognition. The AQUA system, a fluorescence-based system with subcellular compartmentalisation capabilities, has previously been used to quantify biomarkers such as $\boldsymbol{\alpha}$-methylacyl coenzyme A racemase and epidermal growth factor receptor specifically in tumours, but it typically requires the process of masking using other biomarkers,

\begin{tabular}{ll} 
Table 2 Study cohort characteristics & Swedish \\
Watchful Waiting Cohort II: stained for $\alpha$-catenin) \\
\hline No of patients & 411 \\
& Mean age, years \\
Age at diagnosis & $73.93(51-91)$ \\
Gleason score & $n(\%)$ \\
4 & $5(1.6)$ \\
5 & $14(2.5)$ \\
6 & $104(39.2)$ \\
7 & $185(35.6)$ \\
8 & $47(9.3)$ \\
9 & $53(10.3)$ \\
10 & $3(1.6)$ \\
$\%$ Chips with cancer & \\
$<5$ & $74(18.0)$ \\
$5-25$ & $197(47.9)$ \\
$26-50$ & $63(15.3)$ \\
$>50$ & $77(18.7)$ \\
t-Stage & \\
T1a & $74(18.0)$ \\
T1b & $337(82.0)$ \\
Cause of death & \\
PC death & $72(17.5)$ \\
Non-PC death & $272(66.2)$ \\
Still alive & $67(16.3)$ \\
\hline
\end{tabular}

$\mathrm{PC}$, prostate cancer and may not work in all situations. ${ }^{2} 11$ In addition and importantly, cytokeratins mask both normal and neoplastic epithelial tissues, without the capability of differentiating between these compartments. Software that could automate the process of tumour classification and improve tissue segmentation for quantitative scoring would mean significant progress for the analysis of immunohistochemistry. The commercially available Nuance system from CRi (Woburn, Massachusetts, USA) could
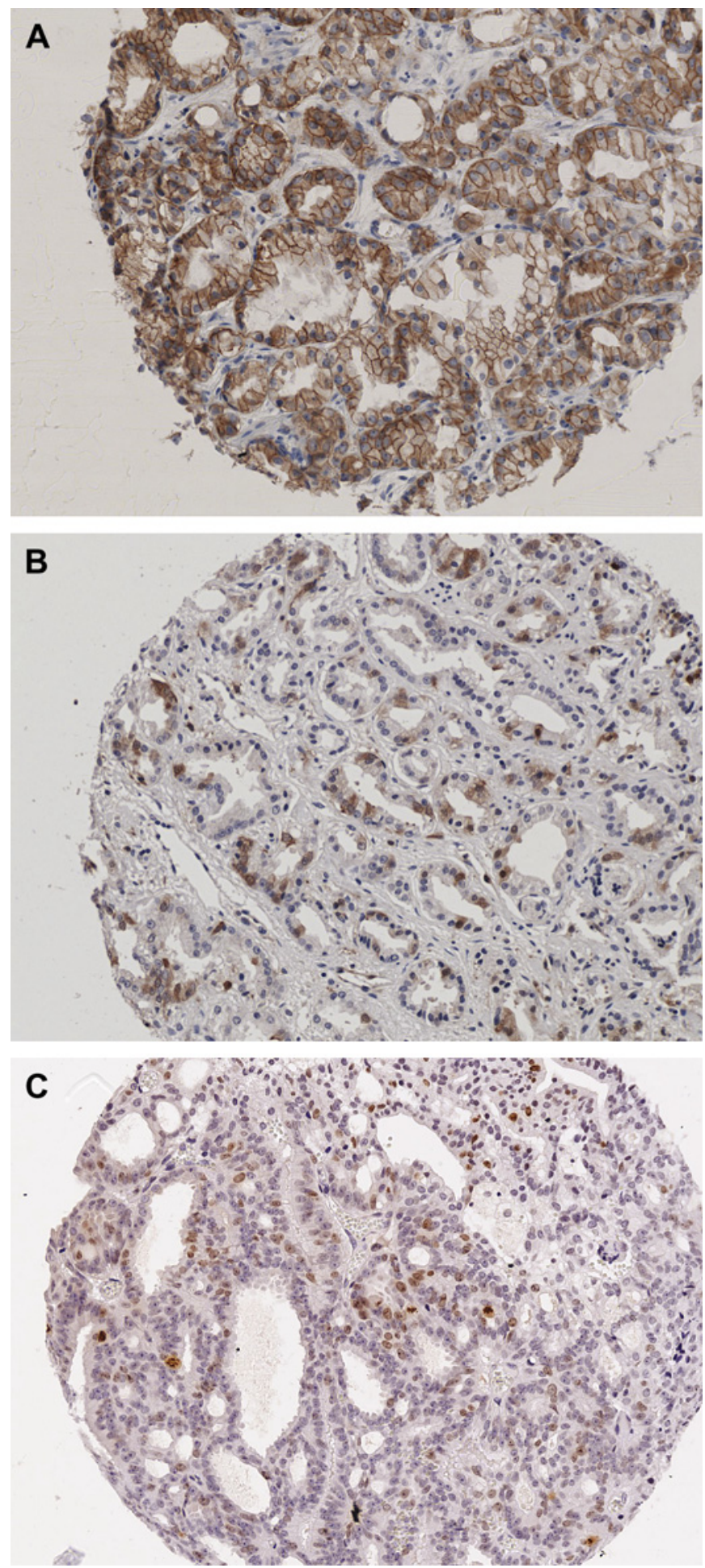

Figure 1 Immunohistochemical staining $(\times 20)$ of prostate adenocarcinoma for membranous marker $\alpha$-catenin $(A)$, nuclear marker Ki-67 (B) and cytoplasmic marker stathmin (C). 
Figure 2 Tissue segmentation: comparison of a raw image (left) with one with tissue classification using inForm (right) for immunohistochemically stained tissue for $\alpha$-catenin (A) and stathmin (B).
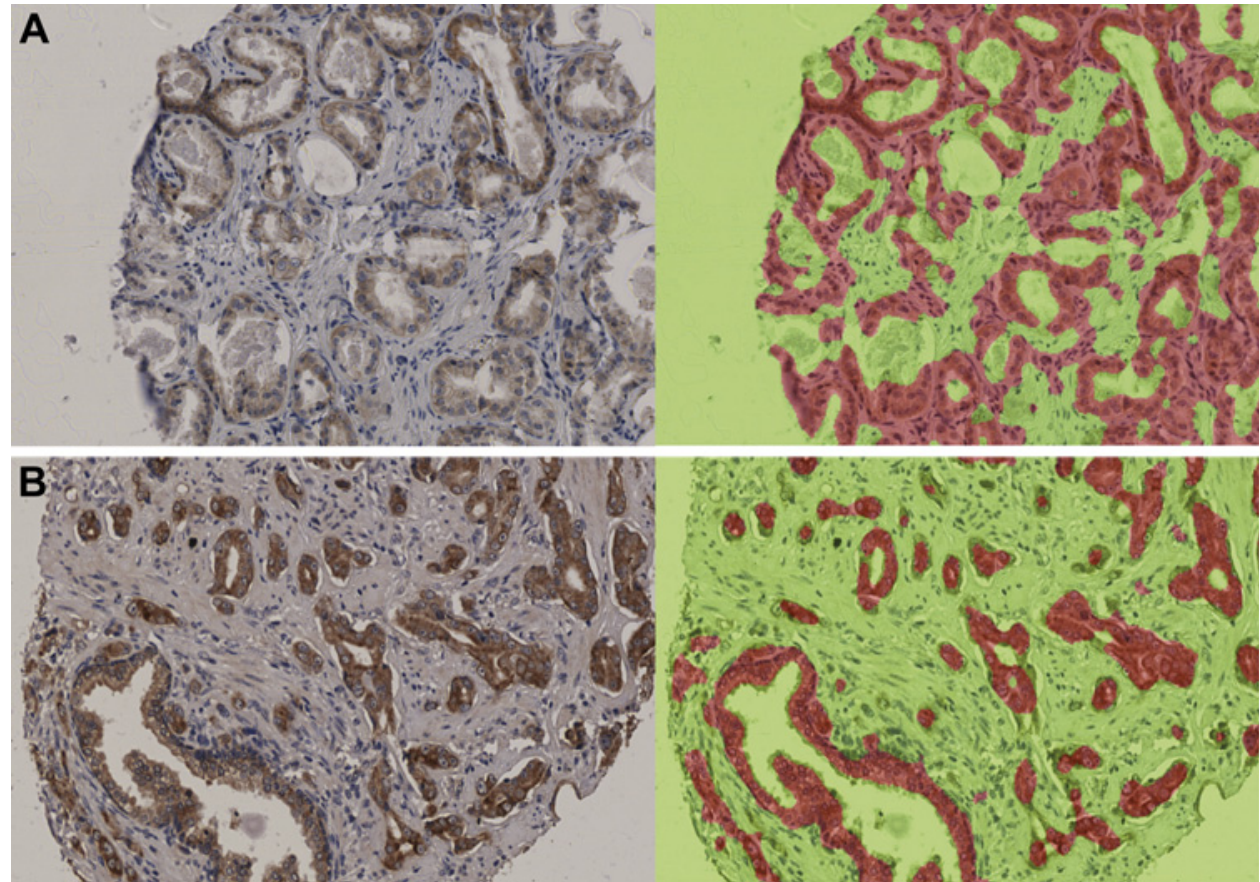
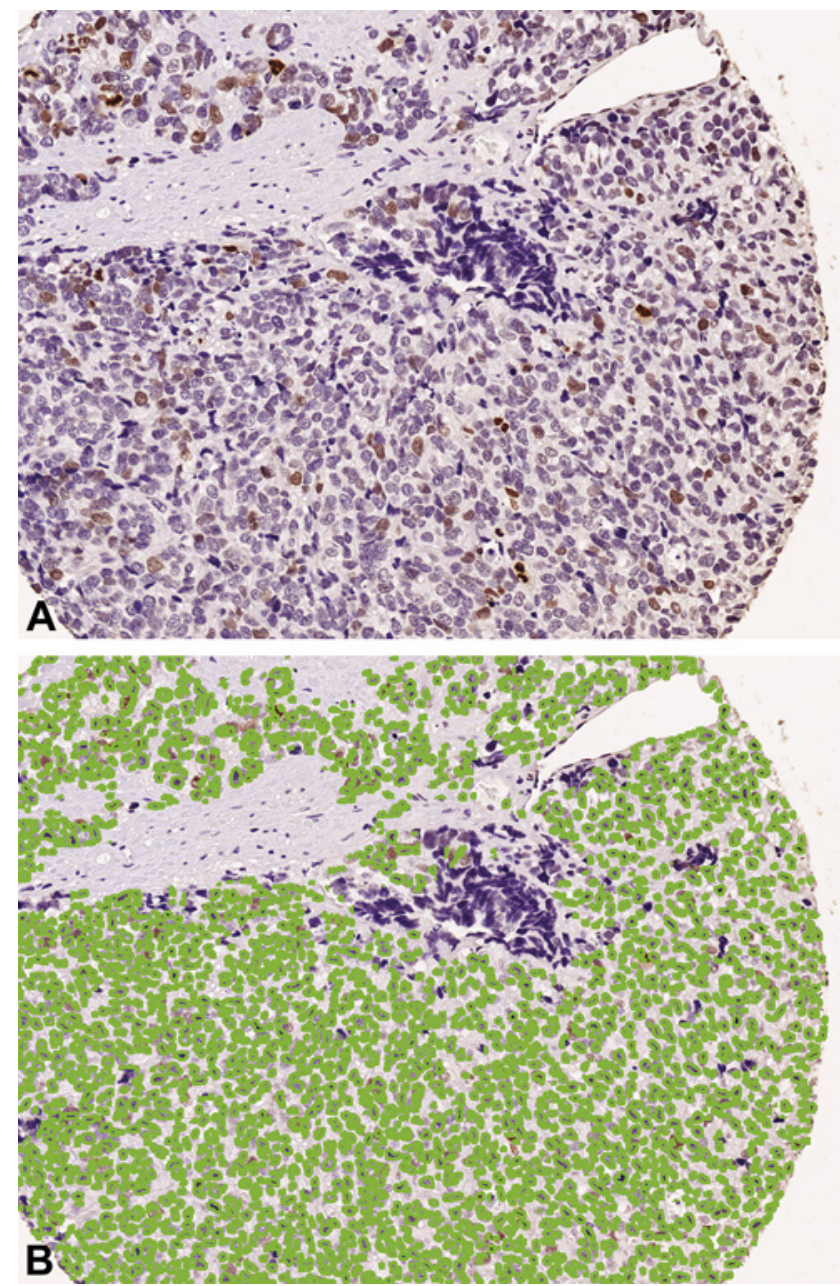

Figure 3 Cell segmentation: inForm classified the nuclei of tissue stained with nuclear marker Ki67-raw image $(A)$ with the classified image (B). The nuclear classification is outlined. potentially help fill this gap, as its multispectral capabilities have the ability to separate visual components to demarcate different cellular components. ${ }^{12}$ In this study we show that automated quantitative analysis of immunohistochemistry on TMA using the Nuance system produces comparable results to the Ariol system. Furthermore, we demonstrate both the added advantages of a multispectral imager for such image analysis and also the potential shortcomings of such a system.

\section{METHODS}

\section{Case selection}

As proof of principle we focused on cases of prostatic adenocarcinoma as these cancers tend to exhibit heterogeneity in terms of morphology. These samples were obtained from the Swedish Watchful Waiting Cohort, which consists of samples from a population-based cohort of men with localised prostate cancer diagnosed incidentally by transurethral resection of the prostate for symptomatic benign prostatic hyperplasia, as previously described. ${ }^{13} 14$ While these samples were collected and immunohistochemically stained primarily for parallel studies, we have used them to compare the image analysis systems as a proof of principle. A total of 571 and 411 cases was represented on five and six TMA for two cohorts, respectively (tables 1 and 2).

\section{Immunohistochemistry}

Four-micrometre sections of each TMA were cut and mounted on glass slides. For antigen unmasking, heat-mediated antigen retrieval was performed on deparaffinised sections by microwaving in citrate buffer $(10 \mathrm{mmol} / \mathrm{l}$ sodium citrate buffer; $\mathrm{pH}$ 6.0) before incubation with primary antibodies. Membranous marker $\alpha$-catenin (mouse monoclonal; 25B1; Abcam, Cambridge, $\mathrm{MA}$ ), cytoplasmic marker stathmin (rabbit polyclonal; Cell Signalling, Danvers, MA) and nuclear marker Ki-67 (rabbit polyclonal; Vectorlab, Burlingame, CA) protein levels were examined using dilutions of 1:200, 1:50 and 1:2000, respectively (figure 1). $\alpha$-Catenin binds to $\beta$-catenin, links the E-cadherin complex to the actin cytoskeleton, and stabilises E-cadherin at the adherens junction. ${ }^{15} 16$ In addition to its role in cell-cell 

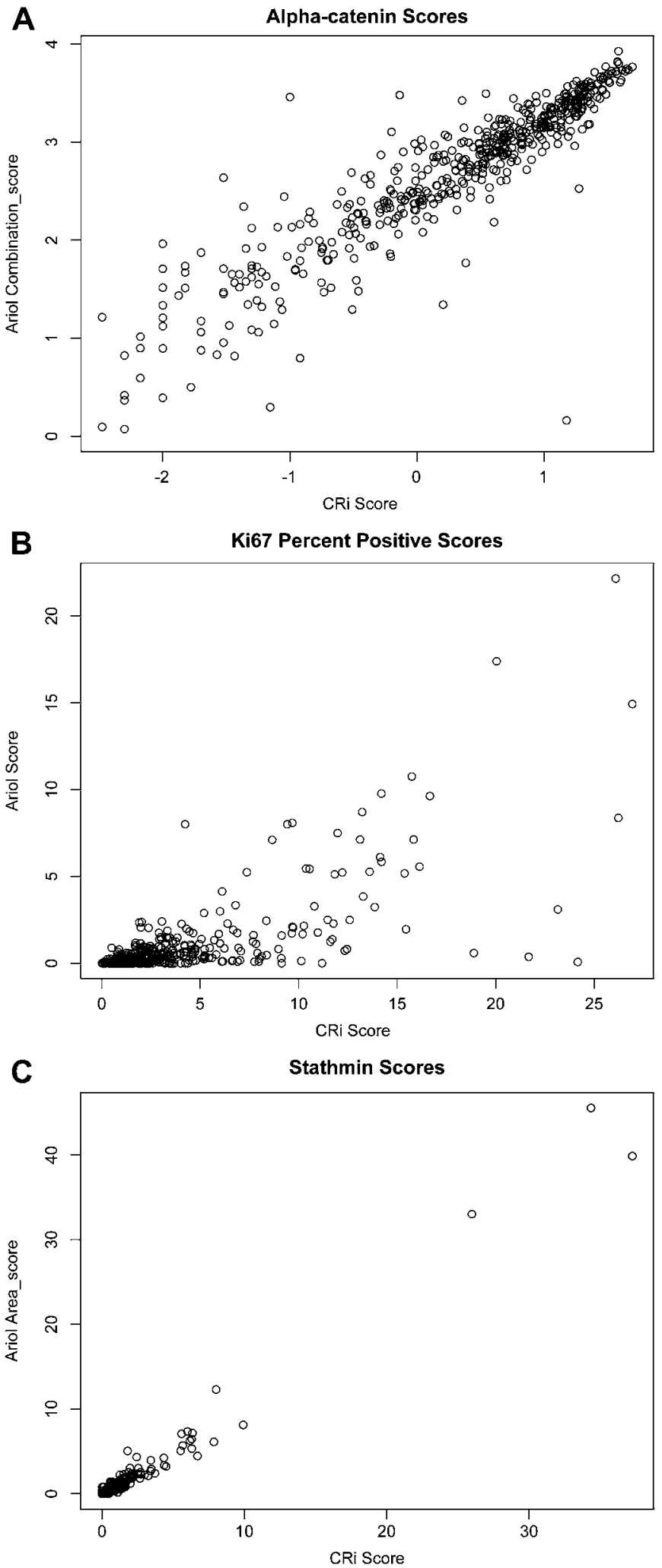

Figure 4 Concordance between immunohistochemical scores generated for each tissue sample by quantitative image analysis using the $\mathrm{CRi}$ system and the Ariol system, shown as dotplots for $\alpha$-catenin (A), stathmin (B) and Ki67 (C). For Ki67, both distributions show a strong clustering at the low end of the spectrum, and a low number of cases with high scores. Based on this, the samples with a score in the $80 \%$ percentile were calculated, and found to overlap with a rate of 0.6824 (those cases positive in one that are also positive in the other: 116/170). The overlap is 0.6486 for the averaged samples (48/74). adhesion, $\alpha$-catenin is also implicated in the regulation of cell proliferation. ${ }^{17-20}$ The microtubule-associated protein stathmin has been implicated as a putative marker of PI3K pathway activation. ${ }^{21-23}$ Haematoxylin was used as a counterstain and diaminobenzadine was used for the immunohistochemical stain. Antibody staining was performed using a streptavidin-horseradish peroxidase system (catalogue nos HK340-9K and HK330-9K; Biogenex, San Ramon, California, USA).

\section{Quantitative image analysis}

\section{CRi Nuance system}

TMA slides were scanned using the CRi Nuance V.2.8 (Woburn) slide scanner following the standard brightfield TMA protocol. The system acquires images at $20 \mathrm{~nm}$ wavelength intervals and combines them into a stack file, which represents one image. This was done automatically to create one image for each core on the TMA. inForm V.0.4.2 software (CRi) was used to analyse the spectral images of each core. Initially, a training set comprising two classes of tissue (that the system would ideally recognise) was created: tumour and other. Representative areas for each of these two classes were marked on 12-16 images from each TMA. The software was trained on these areas using the spectra of both the counterstain (haematoxylin) and the immunostain (diaminobenzadine) and tested to determine how accurately it could differentiate between the two classes (figure 2). The classes ended up being more accurately described as epithelial glandular structures and stroma as manual editing was required to correct tissue classification. This process was repeated until further iterations no longer improved accuracy based on visual inspection of the regions marked by the software.

Histological images were then analysed using the nuclear or cytoplasmic algorithm depending on the antibody. The multispectral imaging capabilities of the Nuance slide scanner allow the software to isolate or segment the nuclei using the unmixed spectra of the nuclear counterstain and the diaminobenzadine immunohistochemical stain used in addition for a nuclear biomarker (figure 3). In turn, cytoplasm was found based on the non-nuclear tumour area. The final score was based on the percentage of the cytoplasmic or nuclear tumour area that was positively stained. A random sample of 30 cores per antibody (corresponding to $5.3 \%$ and $7.3 \%$ of the tissue cores in each cohort, respectively) were reviewed by study participants (CF, $\mathrm{RF}, \mathrm{MF}$ ) to ensure that assigned scores were appropriate. Cores that were difficult to classify (due to technical artifacts such as folds in the tissue, air bubbles, cores overlapping or due to difficulty in morphological classification) were either eliminated from the analysis or manually edited in order to categorise the tissue appropriately.

\section{Ariol SL-50 system}

Corresponding TMA were then scanned and quantitatively scored using the Ariol SL-50 system (Genetix). Scores were generated using the MultiStain assay, providing areas of staining per cell (Area_score), intensity of staining (Intensity_score), and a combination of the two to produce a logarithmic score with an approximately normal distribution (Combination_score). Cytoplasmic and membranous staining was assumed to be proportional to the amount of staining in the whole cell, as these stains showed little non-specific intracellular staining. A Nuclear_score was also calculated based on the percentage of haematoxylinstained nuclei recognised by the Ariol that were positive for diaminobenzadine staining. Areas of tumour were manually identified with masking of the stroma and normal/benign glands from image analysis. 


\section{Time/labour analysis}

Study participant (CF) estimated labour and computer time to scan and analyse the entire Swedish Watchful Waiting II Cohort corresponding to 1200 TMA cores for each stain.

\section{Semiquantitative analysis}

Although human assessment of immunohistochemistry has its limitations it remains the gold standard, so as proof of principle $\boldsymbol{\alpha}$-catenin was scored semiquantitatively, to allow for three-way comparison of manually generated scores with those generated by the automated image analysis systems. One pathologist (NC, blinded to the original diagnosis) scored the sections independently. A modified visual semiquantification method was used using a one-score system for immunointensity as staining for $\alpha$-catenin was homogenous. The semiquantification for immunointensity was scored on a scale of: 0 , negative; 1 , weak; 2 , strong. Scores from all cores from one case were averaged.

\section{Statistical analysis}

Correlation between inForm and Ariol scores and manual semiquantitative scores were calculated using Spearman's correlation coefficient with R V.2.7.0 (R Foundation for Statistical Computing, Vienna, Austria).

\section{RESULTS}

\section{Masking}

The inForm software identified the tumour regions of the tissue with a high degree of accuracy (figure 3). There was, however, a number of cores that required manual editing of the tissue classification mask due to poor tissue quality, benign glands mixing with malignant glands (figure 3 ), or simple mislabelling by the algorithm. These accounted for $10.7 \%$ of $\alpha$-catenin cores (data not shown). Manual editing of the cores was able to correct tissue classification for all downstream analysis. Nuclear and cytoplasmic classification by the inForm software was also found to be accurate for the vast majority of cores analysed (figure 3).

\section{Quantitative image analysis}

The raw image analysis data are presented in figure 4. The correlation between inForm and Ariol scores was dependent on the immunohistochemical stain and was high overall (table 3). The overall correlation between the ranks of the cores using the inForm scores and the Ariol scores for all stains was 0.877 (Spearman's rank correlation; $\mathrm{p}<0.001$ ).

\section{Semiquantitative analysis}

Concordance between manual semiquantitative scores generated for $\alpha$-catenin and quantitative scores generated by the CRi and Ariol systems was $0.591(p<0.0001)$ and $0.589(p<0.0001)$, respectively (Spearman's rank correlation; figure 5).

\section{Time/labour analysis}

Two and a half hours of human time and $15 \mathrm{~h}$ of computer time was required to scan and analyse the Swedish Watchful Waiting II Cohort for each stain using the CRi Nuance system; $7.5 \mathrm{~h}$ of human time and $7.5 \mathrm{~h}$ of computer time was required to scan and analyse each stain of the same cohort using the Ariol SL-50 system.

\section{DISCUSSION}

The Ariol SL-50 system is an established tool for quantitatively scoring immunohistochemistry on TMA. ${ }^{24-26}$ Here we show a strong concordance between scores generated for cytoplasmic and nuclear immunohistochemistry from both the CRi Nuance V.2.8 scanner and the Ariol SL-50 system, illustrating the utility of the Nuance imager and inForm software for quantitatively scoring immunohistochemistry. Ki67 is the only stain that did not have a correlation value above 0.9. It remains to be seen whether this represents a strong improvement or a drawback of the CRi system, as a very high concordance with the Ariol does not necessarily represent total biological accuracy. The high correlation values seen for $\alpha$-catenin for all three scores from the Ariol highlights the ability of the binning score to reflect differences in intensity as well as the percentage of staining, simplifying the scoring of different stains. Furthermore, the good correlation between semiquantitative manual scoring and the Ariol/CRi scores suggests that both systems perform equally well at recapitulating human assessment.

The Nuance system has been used previously for multispectral imaging of immunohistochemistry on archival formalinfixed, paraffin-embedded TMA specimens in order to help distinguish cell types and to quantify tissue biomarkers. ${ }^{27-29}$ The inform software allows for the subclassification of tissues and stratification of cells into nuclear and cytoplasmic compartments in a single step. Other groups have used similar automated methods involving intensity thresholds to quantify staining with software from $\mathrm{CRi}^{29}$ Indeed, inForm offers improvements in a number of areas over RGB (red, green, blue) systems such as the Ariol (box 1), probably reflecting the multispectral imaging capabilities of the Nuance scanner. For brightfield applications, the system collects images along the visible light spectrum at a specified number of intervals (usually every $5-20 \mathrm{~nm}$ of wavelength). It then compiles them into an image file, referred to as a cube, in which every pixel has a spectra associated with it. Spectra representing different chromogens are established and can be 'unmixed' so as to visualise their contributions to the image. This allows for specific colour recognition without the difficulty in deciphering between similar and overlapping chromogens. ${ }^{3}$ A similar approach is utilised for fluorescence-based approaches such as quantum dots. ${ }^{30}$ While we were unable to quantify any improvements in classification, the unmixing of chromogens appears to remove

Table 3 Spearman correlation coefficients between inForm binning scores and Ariol scores (mean intensity, area and combination score)

\begin{tabular}{lllrlll}
\hline Cohort & Immunohistochemical stain & Data & N & Intensity score & Area/nuclear score & Combination score \\
\hline Swedish II & $\alpha$-catenin & Samples & 542 & 0.83 & 0.929 & 0.932 \\
Swedish II & $\alpha$-catenin & Tumour cores & 1123 & 0.821 & 0.953 & 0.954 \\
Swedish I & Ki-67 & Samples & 371 & 0.49 & 0.672 & 0.672 \\
Swedish I & Ki-67 & Tumour cores & 851 & 0.424 & 0.659 & 0.657 \\
Swedish I & Stathmin & Samples & 238 & 0.461 & 0.934 & 0.933 \\
Swedish I & Stathmin & Tumour cores & 583 & 0.437 & 0.929 & 0.928 \\
\hline
\end{tabular}

Note: sample data were generated by taking the mean score from all three cores for each sample.

All correlation values were significant with a $\mathrm{p}$ value $<2.2 \times 10^{-16}$. 


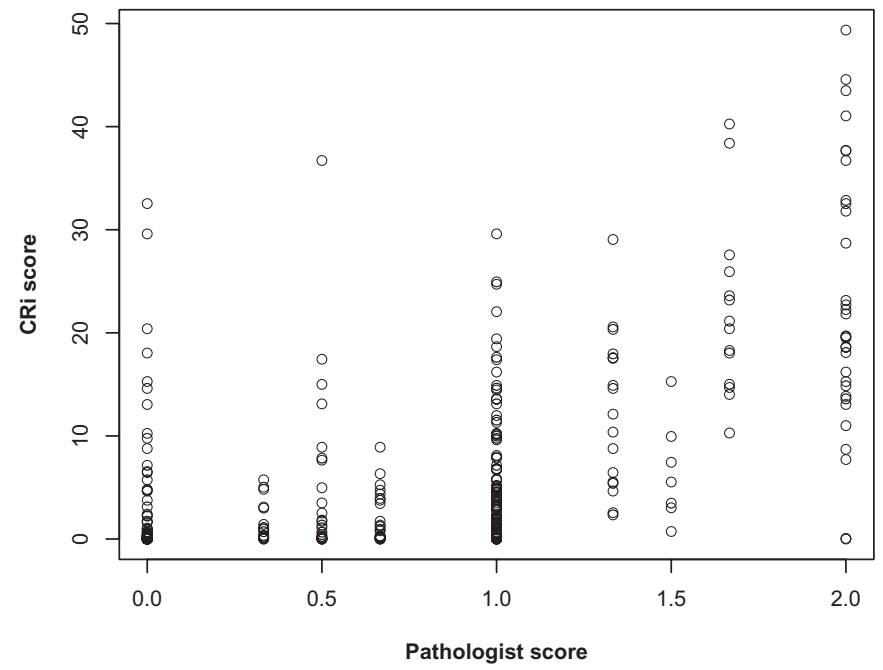

Figure 5 Concordance between immunohistchemical scores generated for Ki67-stained tissue using the CRi system and semiquantitative scores by a pathologist, shown as a dotplot. The concordance between the two scores is good (Spearman's rank correlation 0.591).

background noise, contributing to a more accurate score. Furthermore, the ability to segment nuclear areas with unmixed chromogen spectra (even when diaminobenzadine masks some of the nuclear counterstain) ensures that nuclear staining is not interrogated as cytoplasmic staining. There are, however, a number of disadvantages to the current CRi system. The major limiting factor is the length of time required to scan individual slides: it takes approximately $30 \mathrm{~s}$ per core to scan (an average TMA contained 300-400 cores) following a set-up time of approximately 15-30 min per TMA. In addition, a batch process to analyse the entirety of the image set can take approximately 1 min for every five cores. In contrast, the Ariol system takes approximately $15 \mathrm{~s}$ to scan a core following a similar set-up time. Batch processing on the Ariol takes approximately 1 min per four to five cores. Ultimately, this increased time by the CRi system requires little to no human involvement, and the ability of inForm to classify the tissue automatically can help relieve human labour considerably.

In our study, as the number of TMA slides was relatively small, the time factor was not an overwhelming issue; it may, however, become an issue for study sets containing large numbers of slides on whole tissue sections. Another drawback of the current inForm software is the inability to distinguish between tumour and benign epithelial glandular elements (it

Box 1 List of advantages and disadvantages of the InForm software (CRi) in comparison with the Ariol SL-50 system

inForm advantages

- Specific colour recognition.

- Machine learning classification of stroma from epithelial tissue in prostate.

- Cell by cell information.

inform disadvantages

- Lengthy scans.

- Difficulty with prostatic adenocarcinoma classification.

- Difficulty with variability in tissue appearance within a cohort.

\section{Take-home messages}

The Nuance system from CRi allows for automated analysis of immunohistochemistry using multispectral imaging.

- The scores from the Nuance system and Ariol system are highly correlated, but differences in specific instances could point to functional biology and/or drawbacks in the individual systems.

- There are many factors to consider when choosing an automated scoring system, such as time, labour, reliability and simplicity.

can, however, distinguish between stromal regions and tumour areas). Therefore, during the classification of cores that contain a heterogeneous mix of tissue, the software may mark both tumour and benign prostate as tumour on automated classification, and thus require manual editing of the file to demarcate tumour regions. In this study, the number of cores that required editing varied based on the heterogeneity of cores in a particular cohort and on the staining characteristics of both benign and malignant tissue. The heterogeneity of tissue samples in different cohorts can cause other problems in the analysis. inForm uses 'projects' to help classify different tissues and to organise the analysis of images (it allows up to 16 images per training set per project); this usually equates with one project per immunostain and cohort. In some cohorts there is large variability in the appearance of the tissue, making it difficult for inForm to account for every general tissue appearance. This can lead to a number of cores being misclassified. Splitting up the cohort into smaller projects can help with classification in these scenarios; this will lead to additional set-up time and potentially could lead to scoring bias. Notably for both systems, pathologist input to delineate tumour and normal tissue is essential. While Ariol requires masking on each core to identify tumour, the inForm system can automatically identify glandular tissue that must then be differentiated from tumour manually. We show that whether performed manually for each core as with Ariol or following the recognition of epithelial tissue as with inForm, high concordance rates between scores from the two different systems and with semiquantitative manual scoring can be maintained.

In conclusion, this study suggests a beneficial role for the use of a multispectral imaging system in the automated analysis of immunohistochemistry, and highlights both the inherent advantages and shortcomings of the system when compared with traditional automated and semiquantitative analysis systems.

Acknowledgements The authors would like to thank Edward Stack for his help in assembling images.

Contributors CF collected and analysed data and wrote the paper; DB and XW performed the experiments; SF, MF, NC, NM, SOA, OA and KF collected and analysed data; $\mathrm{ML}$ and RF conceived the idea for the paper, provided guidance and helped write the paper. ML and RF are joint senior authors.

Funding $\mathrm{ML}$ is supported by the Prostate Cancer Foundation, the National Cancer Institute (R01CA131945, P01CA89021 and P50 CA90381), the Linda and Arthur Gelb Center for Translational Research and a gift from Nuclea Biomarkers to the Jimmy Fund and the Loda laboratory. ML is also the recipient of a grant from the Dana Farber Cancer Institute-Novartis Drug Development Program.

Competing interests None.

Provenance and peer review Not commissioned; externally peer reviewed. 


\section{REFERENCES}

1. Jaraj SJ, Camparo $\mathrm{P}$, Boyle $\mathrm{H}$, et al. Intra- and interobserver reproducibility of interpretation of immunohistochemical stains of prostate cancer. Virchows Arch 2009;455:375-81.

2. Rubin MA, Zerkowski MP, Camp RL, et al. Quantitative determination of expression of the prostate cancer protein alpha-methylacyl-CoA racemase using automated quantitative analysis (AQUA): a novel paradigm for automated and continuous biomarker measurements. Am J Pathol 2004:164:831-40.

3. Taylor CR, Levenson RM. Quantification of immunohistochemistry - issues concerning methods, utility and semiquantitative assessment II. Histopathology 2006:49:411-24.

4. Turbin DA, Leung $S$, Cheang MC, et al. Automated quantitative analysis of estrogen receptor expression in breast carcinoma does not differ from expert pathologist scoring: a tissue microarray study of 3,484 cases. Breast Cancer Res Treat 2008;110:417-26.

5. Gokhale S, Rosen D, Sneige N, et al. Assessment of two automated imaging systems in evaluating estrogen receptor status in breast carcinoma. Appl Immunohistochem Mol Morphol 2007;15:451-5.

6. Faratian D, Kay C, Robson T, et al. Automated image analysis for high-throughput quantitative detection of ER and PR expression levels in large-scale clinical studies: the TEAM Trial Experience. Histopathology 2009;55:587-93.

7. Sanders TH, Stokes TH, Moffitt RA, et al. Development of an automatic quantification method for cancer tissue microarray study. Conf Proc IEEE Eng Med Biol Soc 2009;2009:3665-8.

8. Masmoudi H, Hewitt SM, Petrick N, et al. Automated quantitative assessment of HER-2/neu immunohistochemical expression in breast cancer. IEEE Trans Med Imaging 2009;28:916-25.

9. Mulrane L, Rexhepaj E, Penney S, et al. Automated image analysis in histopathology: a valuable tool in medical diagnostics. Expert Rev Mol Diagn 2008;8:707-25.

10. Walker RA. Quantification of immunohistochemistry - issues concerning methods, utility and semiquantitative assessment I. Histopathology 2006:49:406-10.

11. Psyrri A, Yu Z, Weinberger PM, et al. Quantitative determination of nuclear and cytoplasmic epidermal growth factor receptor expression in oropharyngeal squamous cell cancer by using automated quantitative analysis. Clin Cancer Res 2005;11:5856-62.

12. Levenson RM, Fornari A, Loda M. Multispectral imaging and pathology: seeing and doing more. Expert Opin Med Diagn 2008;2:1067-81.

13. Andrén 0, Fall K, Franzén L, et al. How well does the Gleason score predict prostate cancer death? A 20-year followup of a population based cohort in Sweden. J Urol 2006;175:1337-40.

14. Johansson JE, Andrén 0 , Andersson SO, et al. Natural history of early, localized prostate cancer. JAMA 2004;291:2713-19.

15. Kobielak A, Fuchs E. Alpha-catenin: at the junction of intercellular adhesion and actin dynamics. Nat Rev Mol Cell Biol 2004;5:614-25.

16. Scott JA, Yap AS. Cinderella no longer: alpha-catenin steps out of cadherin's shadow. J Cell Sci 2006;119:4599-605.
17. Bullions LC, Notterman DA, Chung LS, et al. Expression of wild-type alpha-catenin protein in cells with a mutant alpha-catenin gene restores both growth regulation and tumor suppressor activities. Mol Cell Biol 1997;17:4501-8.

18. Ewing $\mathbf{C M}, \mathrm{Ru} \mathrm{N}$, Morton RA, et al. Chromosome 5 suppresses tumorigenicity of PC3 prostate cancer cells: correlation with re-expression of alpha-catenin and restoration of E-cadherin function. Cancer Res 1995:55:4813-17.

19. Watabe M, Nagafuchi A, Tsukita S, et al. Induction of polarized cell-cell association and retardation of growth by activation of the E-cadherin-catenin adhesion system in a dispersed carcinoma line. J Cell Biol 1994;127:247-56.

20. Matsubara S, Ozawa M. Expression of alpha-catenin in alpha-catenin-deficient cells results in a reduced proliferation in three-dimensional multicellular spheroids but not in two-dimensional monolayer cultures. Oncogene 2004;23:2694-702.

21. Stolarov J, Chang K, Reiner A, et al. Design of a retroviral-mediated ecdysoneinducible system and its application to the expression profiling of the PTEN tumor suppressor. Proc Natl Acad Sci U S A 2001;98:13043-8.

22. Yu YP, Landsittel $D$, Jing $L$, et al. Gene expression alterations in prostate cancer predicting tumor aggression and preceding development of malignancy. J Clin Oncol 2004;22:2790-9.

23. Saal LH, Johansson P, Holm K, et al. Poor prognosis in carcinoma is associated with a gene expression signature of aberrant PTEN tumor suppressor pathway activity. Proc Natl Acad Sci U S A 2007;104:7564-9.

24. Fiorentino $\mathbf{M}$, Zadra G, Palescandolo $\mathbf{E}$, et al. Overexpression of fatty acid synthase is associated with palmitoylation of Wnt1 and cytoplasmic stabilization of betacatenin in prostate cancer. Lab Invest 2008;88:1340-8.

25. Kleiner HE, Krishnan P, Tubbs J, et al. Tissue microarray analysis of elF4E and its downstream effector proteins in human breast cancer. J Exp Clin Cancer Res 2009;28:5.

26. Turashvili G, Leung $\mathrm{S}$, Turbin D, et al. Inter-observer reproducibility of HER2 immunohistochemical assessment and concordance with fluorescent in situ hybridization (FISH): pathologist assessment compared to quantitative image analysis. BMC cancer 2009;9:165.

27. $\mathbf{X u} \mathbf{X}$, Gimotty PA, Guerry D, et al. Lymphatic invasion revealed by multispectral imaging is common in primary melanomas and associates with prognosis. Hum Pathol 2008;39:901-9.

28. O'Donnell RK, Feldman M, Mick R, et al. Immunohistochemical method identifies lymphovascular invasion in a majority of oral squamous cell carcinomas and discriminates between blood and lymphatic vessel invasion. $J$ Histochem Cytochem 2008;56:803-10

29. Mansoor I, Zalles C, Zahid F, et al. Fine-needle aspiration of follicular adenoma versus parathyroid adenoma: the utility of multispectral imaging in differentiating lesions with subtle cytomorphologic differences. Cancer 2008;114:22-6.

30. Snyder EL, Sandstrom DJ, Law K, et al. c-Jun amplification and overexpression are oncogenic in liposarcoma but not always sufficient to inhibit the adipocytic differentiation programme. J Pathol 2009;218:292-300. 